\title{
Alpha-Particle Angular Distributions of At and Rn Isotopes and Their Relation to Nuclear Structure
}

\author{
P. Schuurmans, ${ }^{1}$ B. Will, ${ }^{2}$ I. Berkes,${ }^{3}$ J. Camps,${ }^{1}$ M. De Jesus,${ }^{3}$ P. De Moor,${ }^{1}$ P. Herzog, ${ }^{2}$ M. Lindroos,${ }^{4}$ R. Paulsen, ${ }^{2}$ \\ N. Severijns, ${ }^{1}$ A. Van Geert, ${ }^{1}$ P. Van Duppen, ${ }^{4}$ L. Vanneste, ${ }^{1}$ and NICOLE and ISOLDE Collaborations \\ ${ }^{1}$ Instituut voor Kern- en Stralingsfysica, K.U. Leuven, Celestijnenlaan 200D, B-3001 Leuven, Belgium \\ ${ }^{2}$ Institut für Strahlen- und Kernphysik, Universität Bonn, Nussallee 14-16, D-53115 Bonn, Germany \\ ${ }^{3}$ Institut de Physique Nucléaire, Université Lyon I, 43 Boulevard du 11 Novembre, F-69621 Villeurbanne, France \\ ${ }^{4}$ CERN, PPE division (ISOLDE), CH-1211 Geneva 23, Switzerland
}

(Received 12 March 1996)

\begin{abstract}
We report on an extensive on-line nuclear orientation study of the angular distribution of $\alpha$ particles emitted in the favored decay of neutron deficient At and Rn nuclei near the $N=126$ shell closure. Surprisingly large anisotropies were observed, showing pronounced changes from one isotope to another. Comparing these data with several theoretical models shows that anisotropic $\alpha$ emission in favored decays from near-spherical nuclei can well be explained within the shell model, implying that it is mainly determined by the structure of the decaying nucleus. [S0031-9007(96)01795-4]

PACS numbers: 23.60.+e, 21.10.Gv, 27.80.+w
\end{abstract}

In the past, the observation of anisotropic $\alpha$ emission from heavy nuclei has often been attributed to the tunneling of the $\alpha$ particle through a deformed Coulomb barrier, thus relating $\alpha$ anisotropies to nuclear deformation [1]. An analogous interpretation is used in sub-barrier fusion reactions involving $\alpha$ particles [2]. In this Letter we present the first unambiguous experimental evidence that anisotropic $\alpha$ emission in favored decays, i.e., in transitions which are (almost) unhindered compared to the ground-state-to-ground-state transitions in neighboring even-even nuclei, is not necessarily related to deformedbarrier penetration.

Already in 1928 the exponential energy dependence of the $\alpha$-decay probability was explained by the tunneling of a preformed $\alpha$ particle through the Coulomb barrier of atomic nuclei [3]. Hill and Wheeler extended this idea and suggested that if the nucleus and consequently the Coulomb barrier is deformed, the tunneling probability becomes direction dependent [4]. Thus anisotropic $\alpha$ emission from an oriented ensemble of deformed nuclei should be observed. Later, a firmer theoretical framework was built [5-7], using the shell modelincluding BCS pairing [8]— to compute the formation probability of the $\alpha$ particle at the nuclear surface and using the WKB approximation [9] to calculate tunneling through the (deformed) Coulomb barrier. The first experimental tests of this theory were performed on prolate deformed actinide nuclei [10]. As predicted, a preferential emission of the $\alpha$ particles along the nuclear symmetry axis was observed. However, at that time the source preparation technique as well as the quality of the detectors available did not allow resolution of the different $\alpha$ transitions in the decays investigated and no farreaching conclusions could be drawn. These problems were solved for the first time when high resolution particle detectors operating at $4.2 \mathrm{~K}$ were combined with ion implantation techniques for sample preparation [11]. This work showed the anisotropies of the favored $\alpha$ decay in the nearly spherical nuclei ${ }^{199,201,203}$ At to be remarkably pronounced and strongly varying. Unfortunately, the interpretation of these data was hampered by the limited knowledge of the deformation of these nuclei. They did, however, trigger a number of new theoretical works on anisotropic $\alpha$-particle emission [12-16]. Comparing the different models with the scarce data available, one is led to the conclusion that theoretically as well as experimentally, the relationship between nuclear deformation and the angular distribution of $\alpha$ particles is not well established. It may be noted here that the $\alpha$ anisotropy is determined exclusively by the higher order partial $\alpha$ waves (i.e., with angular momentum $L \neq 0$ ). The $\alpha$ decay of unoriented nuclei is isotropic in space, and hence experiments to measure the decay rate of unoriented nuclei are insensitive to the different values of angular momentum involved.

In order to gain further insight in the process of $\alpha$-particle emission we have measured the anisotropies for the favored $\alpha$ transitions of the odd nuclei ${ }^{205-209} \mathrm{Rn},{ }^{205-211} \mathrm{At}$, and ${ }^{217} \mathrm{At}$ at the NICOLE low temperature nuclear orientation setup [17] on-line to the ISOLDE mass separator at CERN [18]. The Rn isotopes were produced by a $1 \mathrm{GeV}$ proton beam on a ThC target and ionized in a plasma discharge ion-source equipped with a cooled transfer line. After mass separation the nuclei were implanted at low temperatures (down to $12 \mathrm{mK}$ ) into a magnetized high-purity iron foil mounted in a ${ }^{3} \mathrm{He}-$ ${ }^{4} \mathrm{He}$ dilution refrigerator and subsequently oriented. The ${ }^{205-211}$ At nuclei were produced in situ after the $\beta^{+} / \mathrm{EC}$ decay of the implanted $\mathrm{Rn}$ nuclei and their decay was measured at the same time. The neutron-rich isotope ${ }^{217}$ At was obtained from the beta decay of implanted ${ }^{221} \mathrm{Rn}$ followed by $\alpha$ decay of ${ }^{221} \mathrm{Fr}$. 
The $\alpha$ spectra were measured with three PIN diodes, mounted inside the refrigerator at angles $\theta_{i}=16^{\circ}, 50^{\circ}$, and $84^{\circ}$ with respect to the orientation axis and operated at a temperature of $4.2 \mathrm{~K}$. Conventional Ge detectors recorded the $\gamma$ spectra. The sample temperature was monitored with calibrated ${ }^{57} \mathrm{CoFe}$ and ${ }^{54} \mathrm{MnNi}$ nuclear thermometers. For each $\alpha$ transition, the angular distribution function $W(\theta)$ was calculated from the ratio of the intensities $N(\theta)$ at low temperatures (i.e., $T<100 \mathrm{mK}$; "cold") and at $\approx 1.4 \mathrm{~K}$ (no orientation; "warm"). This function can be written as [19]

$$
W(\theta)=1+f \sum_{k \neq 0} A_{k} B_{k} P_{k}(\cos \theta) .
$$

The factor $f$ represents the effective fraction of nuclei that is oriented by the hyperfine interaction, assuming that the rest $(1-f)$ is not oriented at all. Its magnitude was determined from the anisotropy [i.e., $W\left(\theta_{i}\right) / W\left(\theta_{j}\right)$ versus $1 / T$ ] of well known $\gamma$ transitions. $P_{k}$ are Legendre polynomials, the $Q_{k}$ account for the finite dimensions of source and detector, and the $B_{k}$ parameters describe the orientation. The nuclear information is contained in the directional distribution coefficients $A_{k}$. For $\alpha$ emission they can be written as [20]

$$
A_{k}=\frac{\sum_{L, L^{\prime}} a_{L} a_{L^{\prime}} \cos \left(\sigma_{L}-\sigma_{L^{\prime}}\right) F_{k}^{\alpha}\left(L, L^{\prime}, I_{f}, I_{i}\right)}{\sum_{L} a_{L}^{2}},
$$

where $F_{k}^{\alpha}$ are the Ferentz coefficients modified for $\alpha$ decay [20], and $a_{L}$ and $\sigma_{L}$ are the amplitude and the phase of the $\alpha$ wave with angular momentum $L$. From the $a_{L}$ the mixing ratios $\delta_{0 L} \equiv a_{L} / a_{0}$ are defined. Since $\alpha$ decay is parity conserving, only $L=0,2,4, \ldots$ are involved in the decays investigated in the present work. In the data, evaluation terms with $L$ up to 4 have taken into account.

The experimental anisotropy data for the favored $9 / 2^{-} \rightarrow 9 / 2^{-}$decays of the odd mass nuclei ${ }^{199-211} \mathrm{At}$ and ${ }^{217}$ At are shown in Fig. 1. Note the strong increase in anisotropy with increasing neutron number from $A=199$ to 211 and the fact that the anisotropy of ${ }^{217} \mathrm{At}$, situated beyond the neutron shell closure at $N=126$, is negative again. It is uncertain whether the orientation of ${ }^{217} \mathrm{At}$ was complete due to the short half-life of only $32 \mathrm{~ms}$. Futhermore, some contribution of unoriented ${ }^{217}$ At nuclei that had recoiled out of the iron foil after $\alpha$ decay of ${ }^{221} \mathrm{Fr}$ will have further reduced the anisotropy. Therefore, the observed effect (and, consequently, the deduced $\delta_{02}$ value) has to be regarded as a lower limit for this isotope. It may be noted here that, in spite of different experimental conditions, a previous measurement on ${ }^{217}$ At yielded very similar results [21].

At the lowest temperatures, where orientation saturates, the orientation parameters depend only on the spin of the oriented state and can be calculated exactly. Moreover, since the data are always for the same favored $9 / 2^{-} \rightarrow 9 / 2^{-}$transition, the anisotropy change cannot be attributed to the spin dependent factors in the angular distribution function. Finally, the implantation characteristics are the same for all isotopes since all experiments were performed using cold on-line implantation into a single iron foil and no decrease of the fraction $f=0.810(20)$ with increasing implantation dose could be observed. Hence the only dominant factor the anisotropy changes can be attributed to is a change of the relative amplitude of the $L \neq 0$ partial waves. Experimental mixing ratios $\delta_{0 L}(L=2,4)$ resulting from the anisotropies in Fig. 1 are listed in Table I. Clearly the intensity of the $L=2$ wave [defined as $\left.\delta_{02}^{2} /\left(1+\delta_{02}^{2}+\delta_{04}^{2}\right)\right]$ varies widely with a maximum value of about $4 \%$, while the contribution of the $L=4$ wave is very small.

Anisotropy data for the favored $5 / 2^{-} \rightarrow 5 / 2^{-}$decays of ${ }^{205,207,209} \mathrm{Rn}$ are shown in Fig. 2. The anisotropies are close to each other in this case. Mixing ratios are listed in Table I. The contribution of the $L=2$ wave turns out to be about $1 \%$ for all three nuclei and varies only slightly from one nucleus to another.

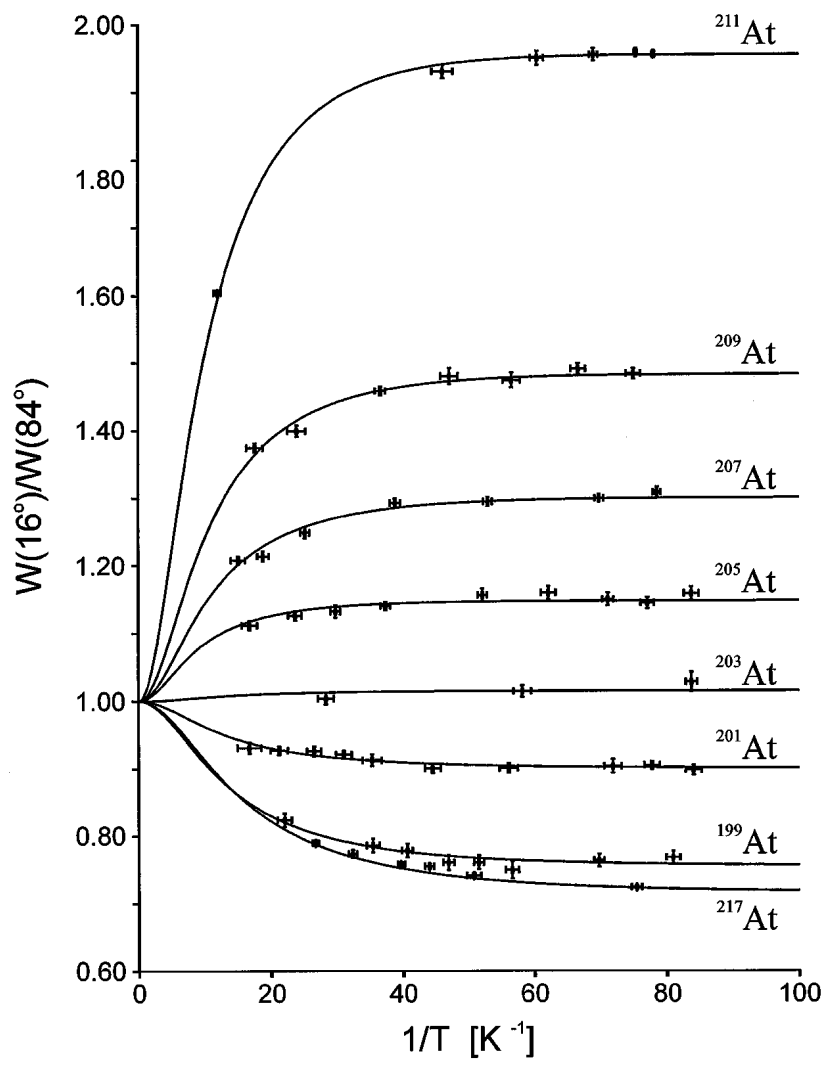

FIG. 1. The experimental $\alpha$ anisotropy $W\left(16^{\circ}\right) / W\left(84^{\circ}\right)$ versus inverse temperature for the favored $\pi h_{9 / 2} \rightarrow \pi h_{9 / 2}$ decays of the odd isotopes ${ }^{199-211}$ At and ${ }^{217}$ At. The data for ${ }^{199-203}$ At are from Ref. [11]. 
TABLE I. Experimental directional distribution coefficients $A_{2}$ and $A_{4}$ and mixing ratios $\delta_{02}$ and $\delta_{04}$ for the favored $\alpha$ transitions of odd At and Rn isotopes. The data for ${ }^{199,201,203}$ At were taken from Refs. [11] and [24] (for these three nuclei $\delta_{04}$ was not determined experimentally).

\begin{tabular}{cccccr}
\hline \hline Isotope & $A_{2}$ & $A_{4}$ & $\delta_{02}$ & $\delta_{04}$ & $L=2(\%)$ \\
\hline${ }^{217} \mathrm{At}$ & $<-0.172(6)$ & $\approx 0$ & $<-0.086$ & $\ldots$ & $>0.73$ \\
${ }^{211} \mathrm{At}$ & $0.400(11)$ & $0.006(18)$ & $0.201(5)$ & $-0.013(10)$ & 3.88 \\
${ }^{209} \mathrm{At}$ & $0.228(7)$ & $-0.033(7)$ & $0.115(4)$ & $-0.024(4)$ & 1.31 \\
${ }^{207} \mathrm{At}$ & $0.153(5)$ & $-0.037(6)$ & $0.077(3)$ & $-0.023(4)$ & 0.59 \\
${ }^{205} \mathrm{At}$ & $0.081(4)$ & $-0.047(7)$ & $0.041(2)$ & $-0.027(4)$ & 0.17 \\
${ }^{203} \mathrm{At}$ & $0.010(6)$ & $\ldots$ & $0.005(3)$ & $\ldots$ & 0.003 \\
${ }^{201} \mathrm{At}$ & $-0.058(4)$ & $\ldots$ & $-0.029(2)$ & $\ldots$ & 0.084 \\
${ }^{199} \mathrm{At}$ & $-0.152(8)$ & $\ldots$ & $-0.076(4)$ & $\ldots$ & 0.57 \\
${ }^{209} \mathrm{Rn}$ & $0.211(6)$ & $-0.05(2)$ & $0.108(3)$ & $-0.033(15)$ & 1.15 \\
${ }^{207} \mathrm{Rn}$ & $0.193(7)$ & $-0.03(3)$ & $0.098(4)$ & $-0.019(15)$ & 0.95 \\
${ }^{205} \mathrm{Rn}$ & $0.177(8)$ & $0.00(2)$ & $0.089(4)$ & $-0.003(14)$ & 0.79 \\
\hline \hline
\end{tabular}

To interpret our data, spherical shell model calculations using the formalism of Mang and Rasmussen [7] were employed [22,23]. The $\alpha$-particle formation amplitude at the nuclear surface was obtained from shell-model wave functions near ${ }^{208} \mathrm{~Pb}$. In the computation of the tunneling probability, only the spherical part of the Coulomb barrier was used, implying that the tunneling of the $\alpha$ particle does not cause any anisotropy in its angular distribution.

For At nuclei $(Z=85)$, besides BCS pairing, also the $p-n$ interaction between the valence neutron holes and protons must be taken into account to reproduce the experimental data. Nuclear shell theory [7] shows that for odd $Z$ nuclei near $N=126$, the major part of the $L=2$ partial $\alpha$ wave arises from the protons in the $\alpha$ particle, which give a positive contribution to the anisotropy. The quadrupole part of the $p-n$ interaction polarizes the core, thus producing a mixed ground state containing $2^{+}$ neutron excitations. The $L=2$ component in the $\alpha$ particle arising from these neutron excitations appears to be

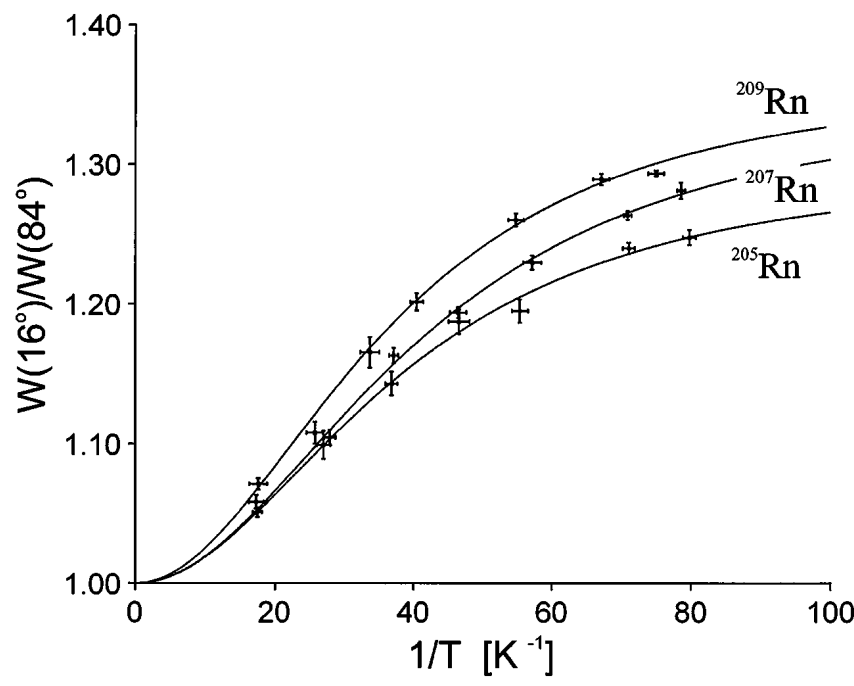

FIG. 2. The observed $\alpha$ anisotropy for the favored $\nu f_{5 / 2} \rightarrow$ $\nu f_{5 / 2}$ decays of ${ }^{205,207,209} \mathrm{Rn}$ versus inverse temperature. negative and its contribution increases with an increasing number of neutron holes (particles) below (above) the $N=126$ shell closure, thus causing a change in anisotropy. The qualitative trend of the At $\alpha$ anisotropies (Fig. 1) agrees with this model as the anisotropy becomes more positive when the number of neutron holes decreases. Furthermore, the approximate linearity of the $\delta_{02}$ values versus the number of neutron holes $N_{\nu}$ for $N<126$ agrees well with the usual parametrization of the $p$ - $n$ quadrupole interaction $Q_{\pi \nu}$ in this region, i.e., $Q_{\pi \nu} \sim N_{\nu}$ [24]. A pure shell model calculation we did for ${ }^{211} \mathrm{At}$ (with $N=126$ ) yielded a theoretical $\delta_{02}$ value of 0.207 , in very good agreement with the experimental value of $0.201(4)$. Finally, the negative $\delta_{02}$ value found for ${ }^{217} \mathrm{At}$ indicates that the proposed mechanism is the same for neutron holes and for neutron particles.

For odd-neutron isotopes near closed shells, such as, e.g., $\mathrm{Rn}$, the $L=2$ part in the $\alpha$ wave is mainly built up by the neutrons in the $\alpha$ particle. In this view, the small increase of the $L=2$ contribution from ${ }^{205} \mathrm{Rn}$ to ${ }^{207} \mathrm{Rn}$ and ${ }^{209} \mathrm{Rn}$ can be explained via the change in the BCS occupation probabilities of the neutron orbitals below $N=$ 126. Indeed, spherical shell model calculations using a BCS pairing strength $G=(25 \mathrm{MeV} / A) \approx 0.120 \mathrm{MeV}$, yielded $\delta_{02}=0.096,0.089$, and 0.081 for ${ }^{209} \mathrm{Rn},{ }^{207} \mathrm{Rn}$, and ${ }^{205} \mathrm{Rn}$, respectively, in good agreement with experiment. Moreover, these values turn out to be fairly insensitive to the particular choice of $G$.

Recently, Delion, Insolia, and Liotta have calculated the partial $\alpha$-wave amplitudes $a_{L}$ (and thus the angular distribution) for both deformed and nearly spherical nuclei $[12,13]$. They adopted the same approach as in older calculations $[5,6]$ but employed a much larger shell model configuration space to compute the formation probabilities. Their calculations indicated that deformation should be the most important factor in modeling the anisotropic $\alpha$ decay and that the angular distribution pattern should reflect the intrinsic shape of the nucleus. A comparison of experimental $\mathrm{Rn}$ mixing ratios with the calculations of 
Delion et al. (Table VII of Ref. [13]) shows that the predicted deformation dependence (as deduced from experimental quadrupole moments) is indeed found, but also that our experimental $\delta$ values are about a factor of two to ten larger than the theoretical ones listed in Table VII of Ref. [13]. For the favored $\alpha$ decay of the neutron deficient At nuclei these calculations predict an increase of the $L=2$ contribution with increasing nuclear deformation (Fig. 2 of Ref. [13]). Interpreting our data in terms of this model would then lead to the unacceptable conclusion that the deformation of the At nuclei investigated increases towards the neutron shell closure, reaching a maximum at $N=126$. Thus deformed-barrier penetration cannot be the dominant factor in the modeling of anisotropic $\alpha$ emission for these nuclei.

Berggren and Olanders [14-16] performed calculations based on an ( $\alpha$ cluster + core) model in which a quadrupole type interaction between an already existing $\alpha$ particle and the core is diagonalized. The anisotropies calculated in this model turn out to be almost independent of deformation except for a narrow range of very small deformations (i.e., $\left|\beta_{2}\right|<0.01$ ), where the dependence is very violent. The significance, however, of the calculations for such small deformations is not yet understood [25]. For the investigated Rn nuclei this model predicts the experimental anisotropy for ${ }^{205} \mathrm{Rn}$ correctly, but fails by over an order of magnitude for ${ }^{207} \mathrm{Rn}$ and ${ }^{209} \mathrm{Rn}$ [16]. For the At nuclei the trend in the observed variation of the $\alpha$ anisotropies can be reproduced, but the calculated magnitudes differ one to several orders of magnitude from the experimental ones (Figs. 2 and 4 of Ref. [15]). It is only in the already mentioned narrow range of very small deformations that the calculated anisotropies are of the same order as the experimental results. The authors of Ref. [14] already suggested that the observed change in $\alpha$ anisotropy for At isotopes is more likely to reflect structural changes in the nuclear states than deformation changes in the mean field.

In conclusion, our experimental data for a series of At and $\mathrm{Rn}$ isotopes reveal surprisingly large $\alpha$ anisotropies for these weakly deformed or nearly spherical nuclei. Comparing our data with recent calculations that were carried out by Delion et al. [12,13] and were based on deformed-barrier penetration shows that the observed anisotropies cannot be explained in a satisfactory way by their model. Secondly, the extreme cluster model of Berggren [14-16] fails to reproduce the magnitudes of the observed anisotropies by at least one order of magnitude, indicating that this model does not yet provide an accurate description of anisotropic $\alpha$ decay for this type of nuclei either. Finally, the spherical shell model (including the $p-n$ interaction) explains the experimental angular distributions rather well, showing very clearly that the observed change in anisotropy for the near-spherical At and $\mathrm{Rn}$ nuclei is due to a change in the nuclear structure.
We thank A. Insolia, T. Berggren, and K. Heyde for stimulating discussions and R. Bouvier, J.P. Hadjout, and P. Schoovaerts for technical assistance. Three of us (P. H., R. P., B. W.) acknowledge financial support by the BMBF Germany Contract No. 06 BN 181. This work was also supported by the Belgian National Fund for Scientific Research (NFWO). N.S. is senior research associate of the NFWO.

[1] F. A. Dilmanian et al., Phys. Rev. Lett. 49, 1909 (1982); D. J. Decman et al., Nucl. Phys. A436, 311 (1985); V.P. Aleshin and F. A. Ivanyuk, Bull. Acad. Sci. USSR Phys. Ser. 54, 45 (1990).

[2] N. Rowley, Nucl. Phys. A538, 205c (1992); A. M. Stefanini et al., Phys. Rev. Lett. 74, 864 (1995), and references therein.

[3] E. U. Condon and R. W. Gurney, Nature (London) 122, 439 (1928); G. Gamow, Z. Phys. 51, 204 (1928).

[4] D. L. Hill and J. A. Wheeler, Phys. Rev. 89, 1102 (1953).

[5] A. Bohr, P. O. Fröman, and B. R. Mottelson, K. Dan. Vidensk. Selsk. Mat.-Fys. Medd. 29, 10 (1955); P. O. Fröman, Mat.-Fys. Skr. Dan. Vidensk. Selsk. 1, 3 (1957).

[6] J. K. Poggenburg, H. J. Mang, and J. O. Rasmussen, Phys. Rev. 181, 1697 (1969); J. O. Rasmussen, Phys. Rev. 115, 1675 (1959).

[7] H.J. Mang and J.O. Rasmussen, Mat.-Fys. Skr. Dan. Vidensk. Selsk. 2, 3 (1962); J. O. Rasmussen, Nucl. Phys. 44, 93 (1963).

[8] J. Bardeen, L. N. Cooper, and J. R. Schrieffer, Phys. Rev. 108, 1175 (1957).

[9] M. A. Preston and R. K. Badhuri, Structure of the Nucleus (Addison-Wesley, New York, 1974), and Ref. [5].

[10] S. H. Hanauer et al., Phys. Rev. 124, 1512 (1961); A. J. Soinski et al., Phys. Rev. C 2, 2379 (1970); A. J. Soinski and D. A. Shirley, Phys. Rev. C 10, 1488 (1974).

[11] J. Wouters et al., Phys. Rev. Lett. 56, 1901 (1986).

[12] D. S. Delion, A. Insolia, and R. J. Liotta, Phys. Rev. C 46, 884 (1992).

[13] D. S. Delion, A. Insolia, and R. J. Liotta, Phys. Rev. C 49, 3024 (1994).

[14] T. Berggren and P. Olanders, Nucl. Phys. A473, 189 (1987); A473, 221 (1987).

[15] T. Berggren, Hyperfine Interact. 75, 401 (1992).

[16] T. Berggren, Phys. Rev. C 50, 2494 (1994).

[17] K. Schlösser et al., Hyperfine Interact. 43, 141 (1988).

[18] E. Kugler et al., Nucl. Instrum. Methods Phys. Res., Sect. B 70, 41 (1992).

[19] Low Temperature Nuclear Orientation, edited by N.J. Stone and H. Postma (North-Holland, Amsterdam, 1986).

[20] K. S. Krane, in Ref. [19], p. 31.

[21] M. Lindroos et al., Hyperfine Interact. 75, 323 (1992).

[22] K. Heyde (private communication).

[23] J. Wouters, Ph.D. thesis, Leuven University, 1989 (unpublished).

[24] K. Heyde et al., Nucl. Phys. A466, 189 (1987).

[25] T. Berggren (private communication). 